\title{
FAM83D associates with high tumor recurrence after liver transplantation involving expansion of $\mathrm{CD}^{4} 4^{+}$carcinoma stem cells
}

\author{
Binyi Lin ${ }^{1,2,3}$, Tianchi Chen ${ }^{1,2}$, Qijun Zhang ${ }^{1,2}$, Xiaoxiao Lu ${ }^{1,2}$, Zhiyun Zheng ${ }^{1,2}$, Jun \\ Ding ${ }^{1,2}$, Jinfeng Liu ${ }^{1,2}$, Zhe Yang ${ }^{1,2,3}$, Lei Geng ${ }^{1,2,3}$, Liming $\mathbf{W u}^{1,2,3}$, Lin Zhou ${ }^{1,2,3}$, \\ Shusen Zheng ${ }^{1,2,3}$ \\ ${ }^{1}$ Division of Hepatobiliary and Pancreatic Surgery, Department of Surgery, The First Affiliated Hospital, Zhejiang University, \\ Hangzhou 310003, China \\ ${ }^{2}$ Key Laboratory of Combined Multi-Organ Transplantation, Ministry of Public Health, Hangzhou 310003, China \\ ${ }^{3}$ Collaborative Innovation Center for Diagnosis and Treatment of Infectious Diseases, Hangzhou 310003, China \\ Correspondence to: Shusen Zheng, email: zyzss@zju.edu.cn \\ Lin Zhou, email: linzhou19@163.com \\ Keywords: FAM83D, carcinoma stem cells, hepatocellular carcinoma, liver transplantation, CD44 \\ Received: April 05, $2016 \quad$ Accepted: October 01,2016 Published: October 18, 2016
}

\section{ABSTRACT}

To investigate the potential oncogene promoting recurrence of hepatocellular carcinoma (HCC) following liver transplantation (LT), throughput RNA sequencing was performed in a subgroup of HCC patients. The up-regulated FAM83D in HCC tissues was found and further verified in 150 patients by real-time PCR and immunohistochemistry. FAM83D overexpression significantly correlated with high HCC recurrence rate following LT and poor HCC characteristics such as high AFP, poor differentiation. Of cancer stem cells (CSCs) markers, CD44 expression was effectively suppressed when FAM83D was knocked down by siRNA. Meanwhile, the siRNA transfected cells suppressed formation of sphere and ability of self-renew. In a xenograft tumorigenesis model, FAM83D knockdown apparently inhibited tumor growth and metastasis. Microarray assays revealed that FAM83D promotes CD44 expression via activating the MAPK, TGF- $\beta$ and Hippo signaling pathways. Furthermore, CD44 knockdown presented reverse effect on above signaling pathways, which suggested that FAM83D was a key activator of loop between CD44 and above signaling pathways. In conclusion, FAM83D promotes HCC recurrence by promoting CD44 expression and CD44+ ${ }^{+}$CSCs malignancy. FAM83D provides a novel therapeutic approach against HCC recurrence after LT.

\section{INTRODUCTION}

Hepatocellular carcinoma (HCC) is the sixth most common malignancy worldwide and the third most frequent cause of cancer deaths [1,2], accounting for more than $70 \%$ of the total liver cancer, and epidemiologic evidence indicates that HCC will still increase medical and economic burden during the next decades [3]. Despite recently significant advances in screening, diagnosis and treatment for $\mathrm{HCC}$, the survival time of a large proportion of HCC patients is significant low and the 5-year rate remains a dismal $12 \%$. In theory, LT could simultaneously remove the tumor and underlying cirrhosis and don't need to consider the level of liver function impairment, which is currently the most effective therapy for HCC. Unfortunately, post-LT HCC recurrence and metastasis greatly give rise to the unsatisfactory long-term survival following LT. Furthermore, the number of HCC patients on the waiting line for LT and out of LT criteria is much more than liver recipients. Although several target drugs are under development, only sorafenib presented a finite survival benefits for individuals with advanced HCC [4]. Therefore, it is essential to find a potentially novel therapeutic target and predicting biomarker of diagnosis, treatment response and recurrence for improving $\mathrm{HCC}$ prognosis. 
FAM83D that belongs to family with sequence similarity 83 genes locates on 20q. Amplification of chromosome $20 \mathrm{q}$ is frequently presented in various cancers and correlated with poor prognosis due to the ability of promoting cancer progression and metastasis [5]. Expectedly, several prior studies suggested that FAM83D is overexpressed in a variety of cancers including HCC, metastatic lung adenocarcinomas and ovarian cancer as a growth promoting gene [6-8]. A proteomic analysis of the human spindle apparatus revealed that FAM83D localizes to spindle microtubules regulating distribution of sister chromatids [9]. Furthermore, FAM83D coordinates chromokinesin Kid locating in spindle and generating polar ejection and chromosome congression [10]. Deficiency of FAM83D courses malfunctioning of the spindle leading to chromosome missegregation and aneuploidy, a hallmark of cancer. FAM83 protein family only contain a domain (DUf1669) with weak homology to a phospholipase D, and the elevated FAM83 member expression in tumor increase phospholipase D activity resulting in hyperactivation of epidermal growth factor receptor (EGFR) [11]. Cytoplasmic FAM83D interact with CRAF and promote CRAF translocation leading to activation of MAPK signaling spathways [12]. However, FAM83D whether associates with HCC recurrence after LT remains unclear, and the mechanism of FAM83D promoting HCC progress need to be further clarified. In the study, we found that FAM83D overexpression correlated with up-regulated CD44 expression.

$\mathrm{HCC}$ tissues are maintained in a hierarchical organization of heterogeneous groups of cancer cells including rare cancer stem cells (CSCs) with the ability of self-renewal and differentiation [13]. The existence of CSCs were verified in the context of various solid cancers and considered as one mechanism accounting for tumor growth and metastatic activity [14], and chemoresistance and recurrence $[15,16]$. The circular CSCs and extra-hepatic disseminated CSCs are able to survive for many years in a dormant state benefiting from their high resistance ability, and result in tumor relapse and renewed aggression after LT. Several prior studies identified CD133, CD44, CD24, CD90, CD13 and EpCAM as potential candidate CSC markers in HCC [17-22]. CD44 is a member of the cartilage link protein family and an important receptor of hyaluronan (HA). Alternative splicing could insert up to ten variant exon products into between domains 5and 6 leading to several CD44 variants. The crosstalk of CD44s or CD44v with tumor environment amplify a variety of signals endowing CSCs with special properties, such as epithelial-mesenchymal transition (EMT) and high resistance to apoptosis and niche preparation [22].

In the present study, we evaluated the effect of upregulated FAM83D on HCC initiation, progression and recurrence and verified that FAM83D promotes CD44 overexpression via activating MAPK, TGF- $\beta$ and Hippo signaling pathways.

\section{RESULTS}

\section{The pattern and significance of FAM83D expression in $\mathrm{HCC}$}

We profiled mRNA expression in liver samples from $10 \mathrm{HCC}$ and corresponding non-HCC tissues (no pre-LT therapy) with RNA sequence and found that 321 upregulated genes and 223 down-regulated genes ( $>2$ folds, $\mathrm{P}<0.01)$. By pathway analysis, we defined the MAPK pathways is the most important in HCC initiation and development (Figure 1 in supplementary file). Among the 544 genes, FAM83D was increased in 8/10 HCC tissues compared with adjacent liver tissues. Additionally, we expansively detected the FAM83D expression in 150 pair specimens with real-time PCR and immunohistochemistry (IHC), and verified FAM83D was apparently up-regulated in HCC tissues (Figure 1A-1C). Moreover, overexpression of FAM83D in HCC was significantly correlated with poor tumor characteristics, i.e. poor differentiation, portal vein tumor thrombus (PVTT), tumor number, and greatest tumor diameter (Figure 1D-1H). Subsequently, KaplanMeier's analysis disclosed that patients with higher FAM83D developed more frequent HCC recurrence (Figure 2A) and presented poorer TFS outcomes after LT. The post-LT 5 years tumor-free survival rate was $75.4 \%$ in FAM83D-low group and $57.1 \%$ in the FAM83Dhigh group respectively. Besides, FAM83D-low patients showed better TFS no matter who was within or beyond LT Criteria of our center (Figure 2B). Cox's proportional hazards regression analysis revealed that high FAM83D expression was an independent predictor of prognosis of LT for HCC (TFS: hazard ratio [HR] 2.30; 95\% confidence interval [CI] 1.15-4.60; P 0.019; Table 1).

\section{FAM83D drives CD44 overexpression in HCC}

Given that CSCs played an important role in $\mathrm{HCC}$ progression and recurrence following LT and the high FAM83D expression in HCC positively correlated with AFP levels, we tested the relationship between FAM83D expression and CSCs biomarkers. A siRNA for FAM83D was transfected into SMMC-7721 and SK-Hep-1 cells with high FAM83D expression, and then the change of CSCs biomarkers expression were defined by RT-PCR. The results verified that FAM83D knockdown significantly decreased the CD44 expression (Figure 3A). Furthermore, in $150 \mathrm{HCC}$ tissues, CD44 expression in the FAM83D high group was apparently higher than the FAM83D low group (Figure 3B). The lentivirus delivery of siRNA for FAM83D was used to achieve FAM83D knock-down HCC cell lines. Expression levels of FAM83D and CD44 were 
markedly down-regulated concurrently. SMMC-7721 and SK-hep-1 presented a 100\% proportion of cells expressing CD44, FAM83D knockdown did not made a decrease in proportion of CD44 positive cells, but flow cytometric analysis (FCA) confirmed that enforced FAM83D knockdown lead to a significantly decrease in fluorescence intensity of CD44 expression (Figure 3C, 3D), and the result was further verified by western blotting (Figure 3E, $3 F)$. In addition, the effect of FAM83D on CD44 variants expression was also detected, and confirming FAM83D knockdown apparently reduced the all of CD44 variants expression (Figure 3G). To further explore the effect of FAM83D on CD44 positive cells expansion, MHCCLM3, Huh7 and HepG2 were transfected with siRNA for FAM83D, the results suggested that FAM83D knockdown not only decreased the fluorescence intensity but also could reduce the proportion of cells expressing CD44 (Figure 3H).
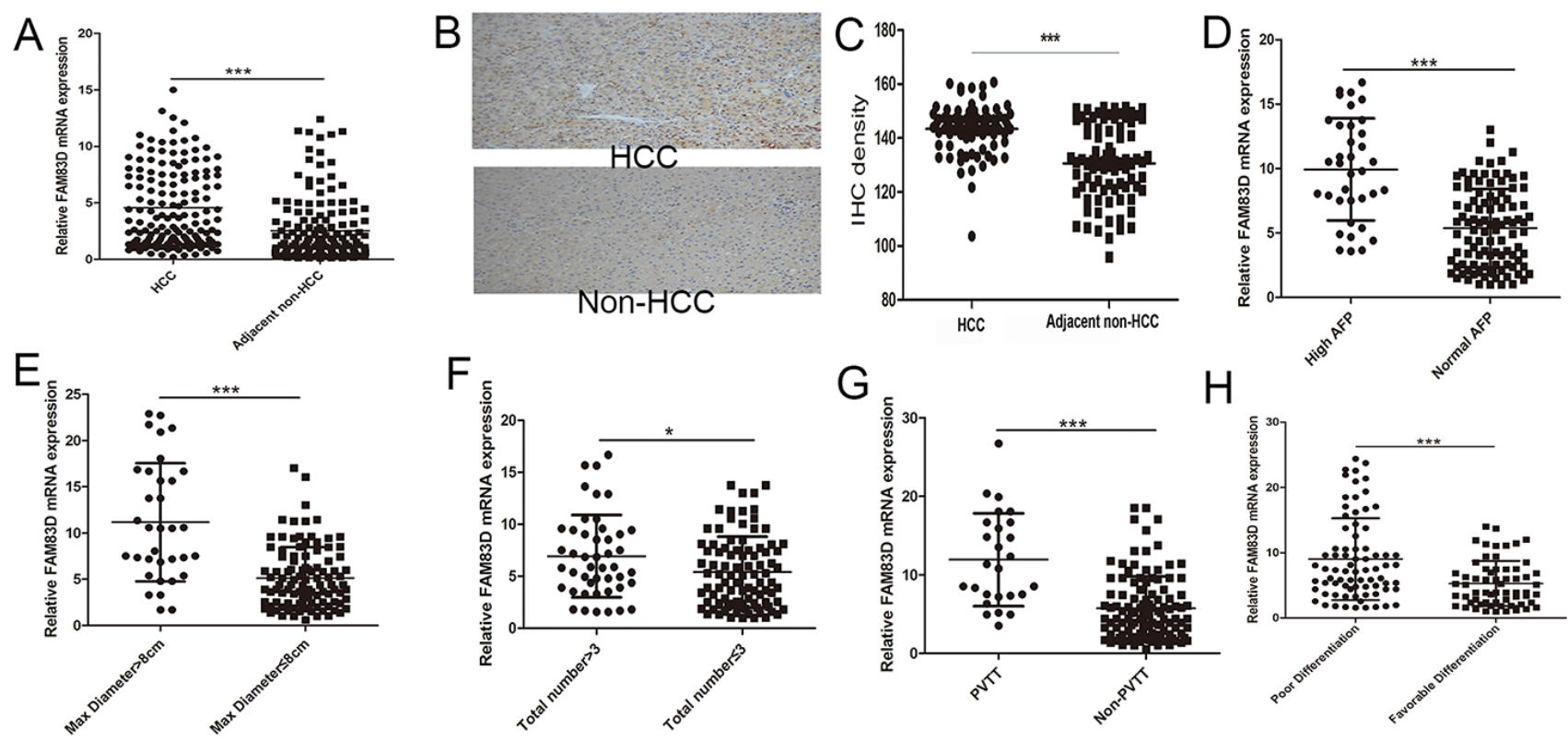

$\mathrm{H}$

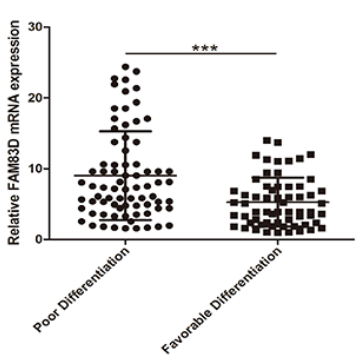

Figure 1: In 150 pair specimens, FAM83D was significantly overexpressed in HCC tissues comparing to adjacent nonHCC tissues A. by RT-PCR. This result was further confirmed by IHC B, C. Furthermore, further overexpression of FAM83D was found in tumors with higher AFP level D., greater tumor diameter E., more tumor number $\mathbf{F}$., portal vein tumor thrombus $\mathbf{G}$. and poorer differentiation $\mathbf{H}$.
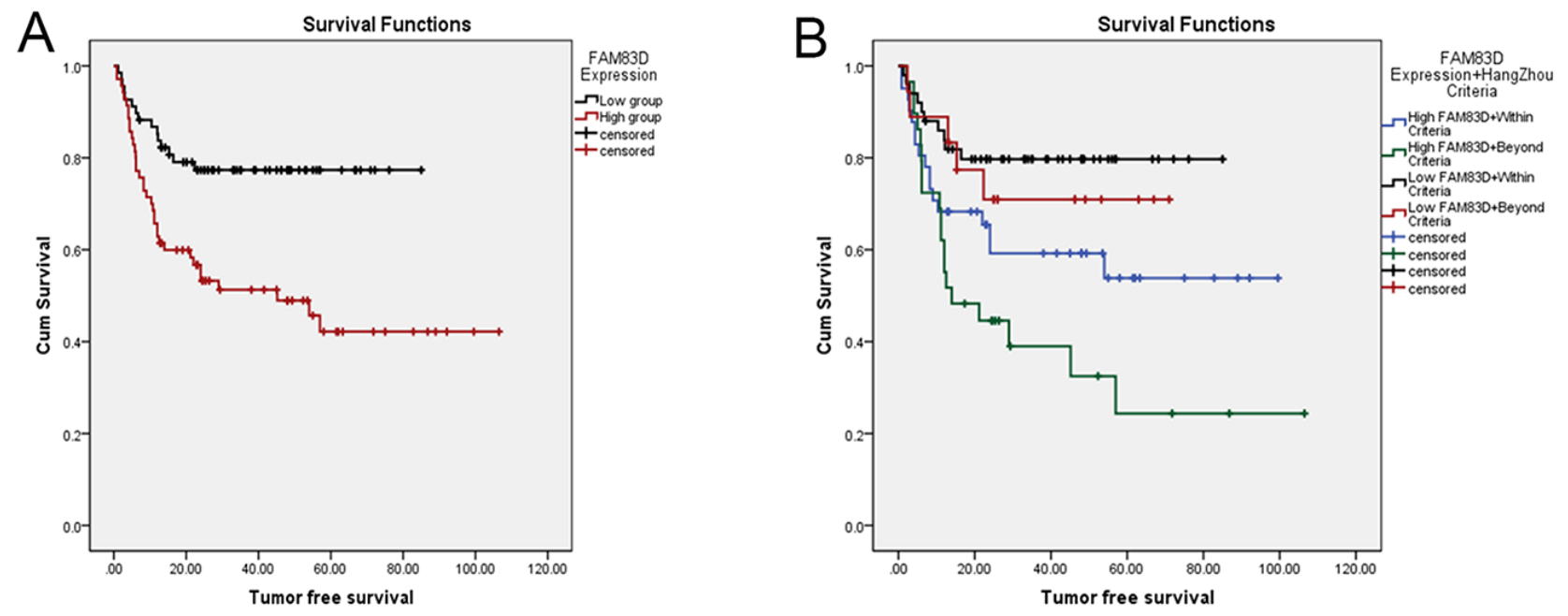

Figure 2: Kaplan-Meier's analysis disclosed that patients with high FAM83D expression developed more frequent HCC recurrence than low FAM83D expression A. In addition, HCC patients with low FAM83D expression maintained the advantage of tumor free survival no matter who was within or beyond LT Criteria $\mathbf{B}$. 
Table 1: Predictors of HCC recurrence following LT

\begin{tabular}{lcccc}
\hline & \multicolumn{2}{c}{ Univariate analyses } & Cox proportional hazards regression \\
\hline Variables & P value & HR $(95 \% \mathrm{CI})$ & P value & $\mathrm{HR}(95 \% \mathrm{CI}) 0.017$ \\
Number & 0.017 & $1.980(1.131-3.465)$ & 0.625 & $1.163(0.634-2.136)$ \\
Diameter $>8 \mathrm{~cm}$ & $<0.001$ & $3.31(1.903-5.711)$ & 0.032 & $2.129(1.067-4.248)$ \\
AFP & 0.003 & $2.299(1.233-3.963)$ & 0.667 & $0.861(0.434-1.706)$ \\
PVTT & $<0.001$ & $3.676(2.118-6.378)$ & 0.010 & $2.265(1.213-4.23)$ \\
Differentiation & 0.003 & $2.557(1.385-4.720)$ & 0.316 & $1.482(0.687-3.19)$ \\
FAM83D & 0.001 & $2.773(1.524-5.047)$ & 0.019 & $2.3(1.15-4.6)$ \\
\hline
\end{tabular}

Note, HR, risk ratio, 95\% CI 95\% confidence interval

\section{FAM83D knockdown decreases stemness}

Several previous published studies identified CD44 is a precise maker of CSC in HCC [22]. o clarify whether FAM83D knockdown inhibits HCC stemness, the spheroid formation assay in vitro that is a classic method to acquire CSCs [23] was performed. Suspension-cultured HCC cells with FAM83D knockdown had lesser and smaller tumor spheroids than controls (Figure 4A, 4B). Furthermore, the HCC cells spheroids were detached and re-suspended to form second spheroid in CSCs culture medium. The second formed spheroids were larger than first generation of spheroids, and FAM83D knockdown groups also presented smaller and lesser spheroids compared to NC groups (Figure 4C). Moreover, the AFP and albumin expression detection revealed that decreased FAM83D expression inhibited the differentiation of CSCs (Figure 4D). Besides, carboxyfluorescein diacetate, succinimidyl ester (CFSE) labeled cells verified FAM83D knockdown significantly reduced the self-renew after suspensionculture (Figure 4E, 4F). These results revealed that FAM83D knockdown effectively inhibited the stemness of CD44 positive CSCs.

\section{FAM83D knockdown inhibits tumorigenesis and metastasis of $\mathrm{HCC}$ in vitro and vivo}

The ability of tumorigenesis and tumor metastasis indicated the stemness of HCC cells with CD44 expression. We investigated the effect of FAM83D on tumor growth and metastasis in nude mice. FAM83DsiRNA-transfected SMMC-7721 cells and negative control cells were subcutaneously injected into nude mice (ten animals per group). Half of mice were sacrificed and the tumors were dissected after one month, remaining half of mice were sacrificed for observing the number of metastasis in lung after 1.5 month. Compared with the controls, FAM83D knockdown presented an apparent decrease in tumor size and weight (Figure 5A). The tumors were stained with CD44. Expectedly, tumors formed by FAM83D-siRNA-transfected SMMC-7721 presented decreased CD44 expression compared with controls (Figure 5B). Furthermore, at least one metastasis with high CD44 expression in lung was observed in the two mice of control group, but there was no pulmonary metastasis in the FAM83D knockdown group (Figure 5C). To further verify the effect of FAM83D on HCC cells colonizing in lung, we established a pulmonary metastasis tumor model using tail vein injection of HCC cells. Consistently, we found that FAM83D knockdown could inhibit tumor colonization in lung. In the control group, four of five mice presented multiple tumor colonies, but only two mice showed one to two tumor colonies in the FAM83D knockdown group (Figure 5D). Besides, the transwell assay confirmed that knockdown of FAM83D would impact migration and invasion of $\mathrm{HCC}$ cell lines in vitro (Figure 5E).

\section{FAM83D regulates multiple CSCs associated signaling pathways}

Gene expression microarray was used to reveal the mechanism underlying FAM83D driving expansion of CD44 positive CSCs. SMMC-7721 cells transfected with siRNA-FAM83D resulted in a total of 1565 differentially expressed genes including 686 up-regulated genes and 879 down-regulated genes comparing with control cells. The data were further analyzed using an online database (http://david.abcc.ncifcrf.gov/) [24]. The gene ontology analysis disclosed that FAM83D knockdown made a deeply effect on multiple biological process including cell migration, differentiation, adhesion (Figure 6A). Furthermore, the Kyoto Encyclopedia of Genes and Genomes (KEGG) analysis indicated that FAM83D could regulate TGF- $\beta$, MAPK, Hippo signaling pathways (Figure 6B). By western blot, we verified that FAM83D knockdown reduced the levels of smad 2 phosphorylation, ERK1/2 phosphorylation, decreased the YAP expression 

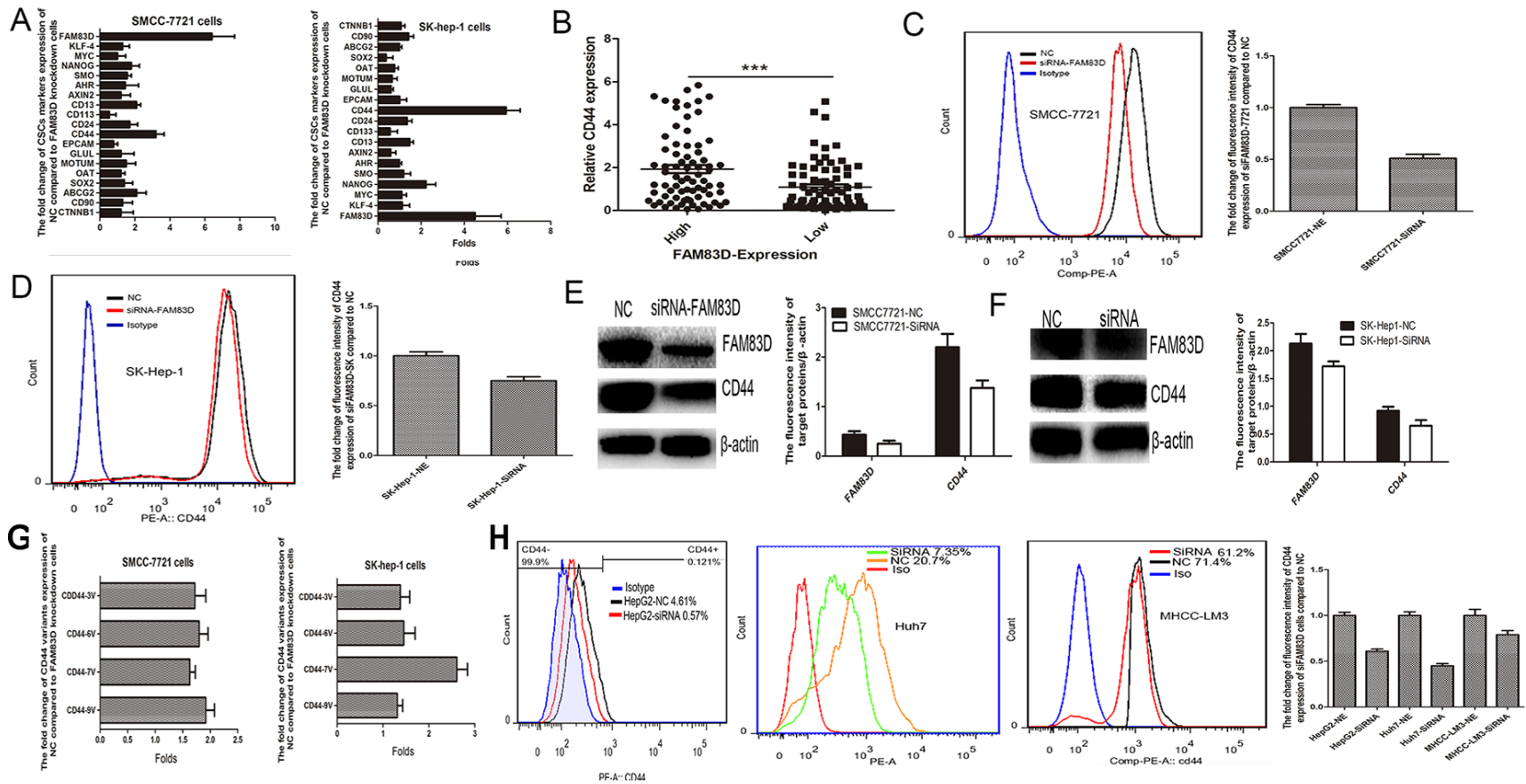

Figure 3: By RT-PCR, all of CSCs biomarkers, CD44 presented the greatest down-regulated folds when FAM83D expression was inhibited in SMMC7721 and SK-Hep-1 A. Furthermore, in the HCC patients with high FAM83D expression, the CD44 expression was apparently up-regulated B. FCA showed that enforced FAM83D knock-down significantly decreased the fluorescence intensity of CD44 expression C, D., and the result was further verified by western blot E, F. Furthermore, FAM83D knockdown apparently reduced the all of CD44 variants expression G. In HepG2, Huh7 and MHCC-LM3 cells, FAM83D knockdown not only decreased the fluorescence intensity, but also could reduce the proportion of cells expressing CD44 $\mathbf{H}$.
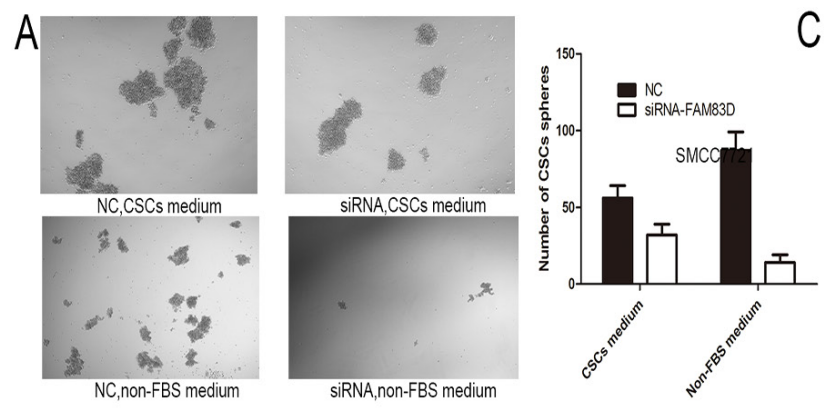

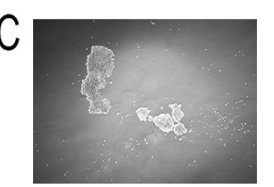

SMCC7721-NC

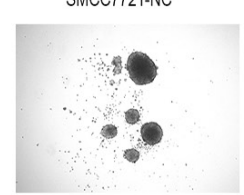

MHCC-LM3-NC

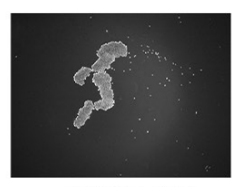

SMCC7721-SiRNA

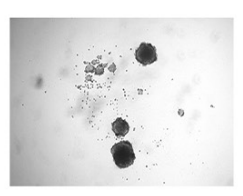

MHCC-LM3-SiRNA

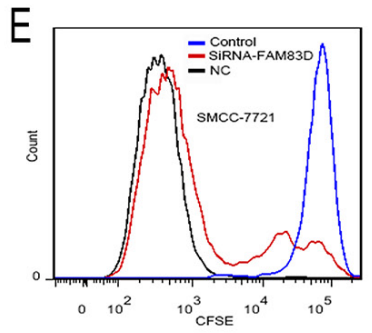

B

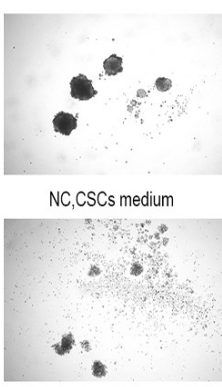

$\mathrm{NC}$, non-FBS medium
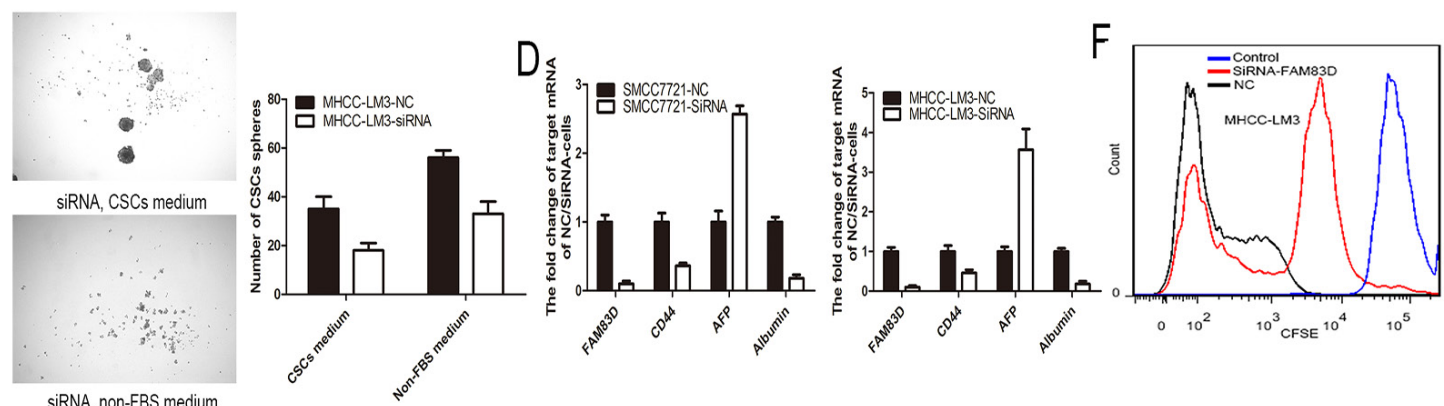

Figure 4: Suspension-cultured SMCC7721 and MHCC-LM3 cells with FAM83D knockdown presented lesser and smaller tumor spheroids than controls no matter in the non-FBS medium or CSCs medium A-B. Furthermore, the second formed spheroids were larger than first generation of spheroids, and FAM83D knockdown groups also presented smaller and lesser spheroids compared to NC groups $\mathbf{C}$. Besides, decreased FAM83D expression increased the AFP expression and decreased the albumin expression in CSCs D. CFSE was used to trace cells and confirmed that FAM83D knockdown cells presented higher concentration of CFSE compared to $\mathrm{NC}$ after suspension-culture E-F. 

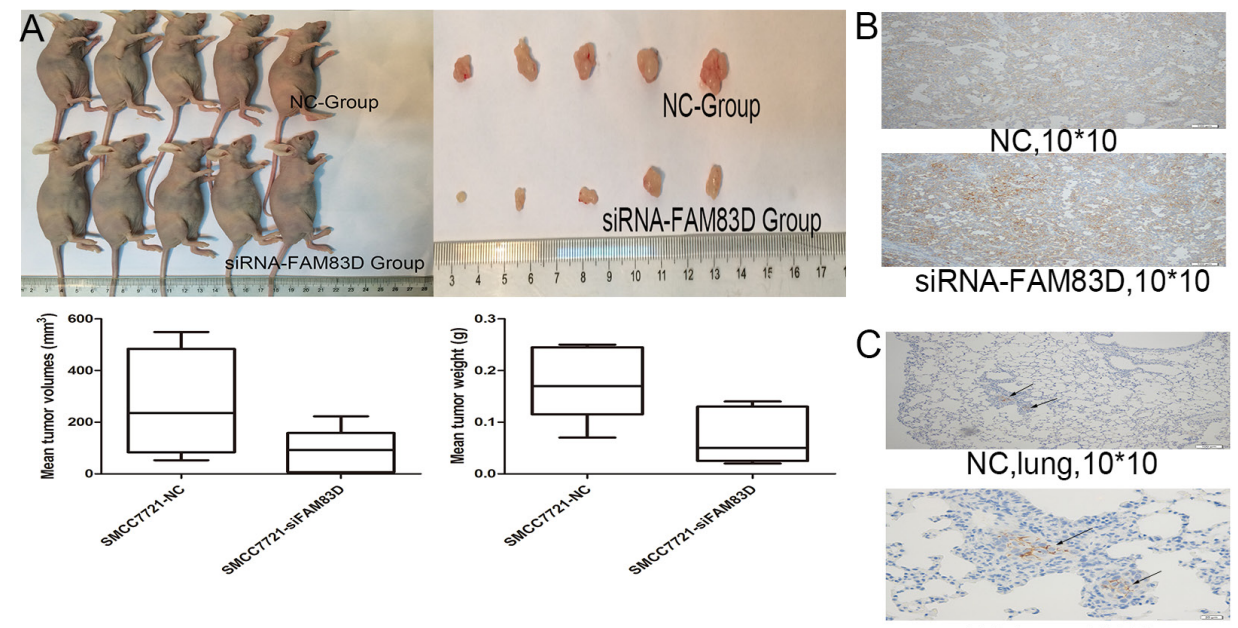

NC,lung, $40 * 10$
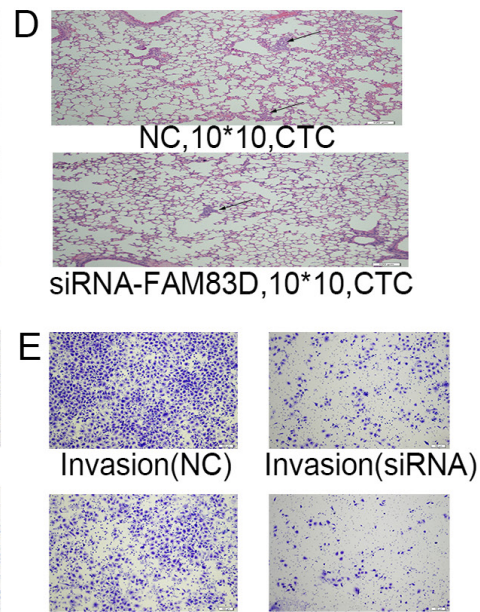

Invasion(NC)

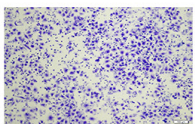

Migration(NC)

Migration(siRNA)

Figure 5: In vivo, FAM83D knockdown inhibited the tumorigenicity, and FAM83D-siRNA transfected SMMC-7721 cells presented an apparent decrease in tumor size and weight comparing the control cells A. Expectedly, FAM83D knockdown also reduced the CD44 expression in subcutaneously formed tumor tissues B. In NC group, at least one metastasis with high CD44 expression in lung was observed, but there was no pulmonary metastasis in the FAM83D knockdown group C. In the CTC model, the NC group presented more tumor colonies in lung than the FAM83D knockdown group D. Furthermore, in vitro, the transwell assay confirmed that knockdown of FAM83D would impact migration and invasion of SMCC7721 cells E.
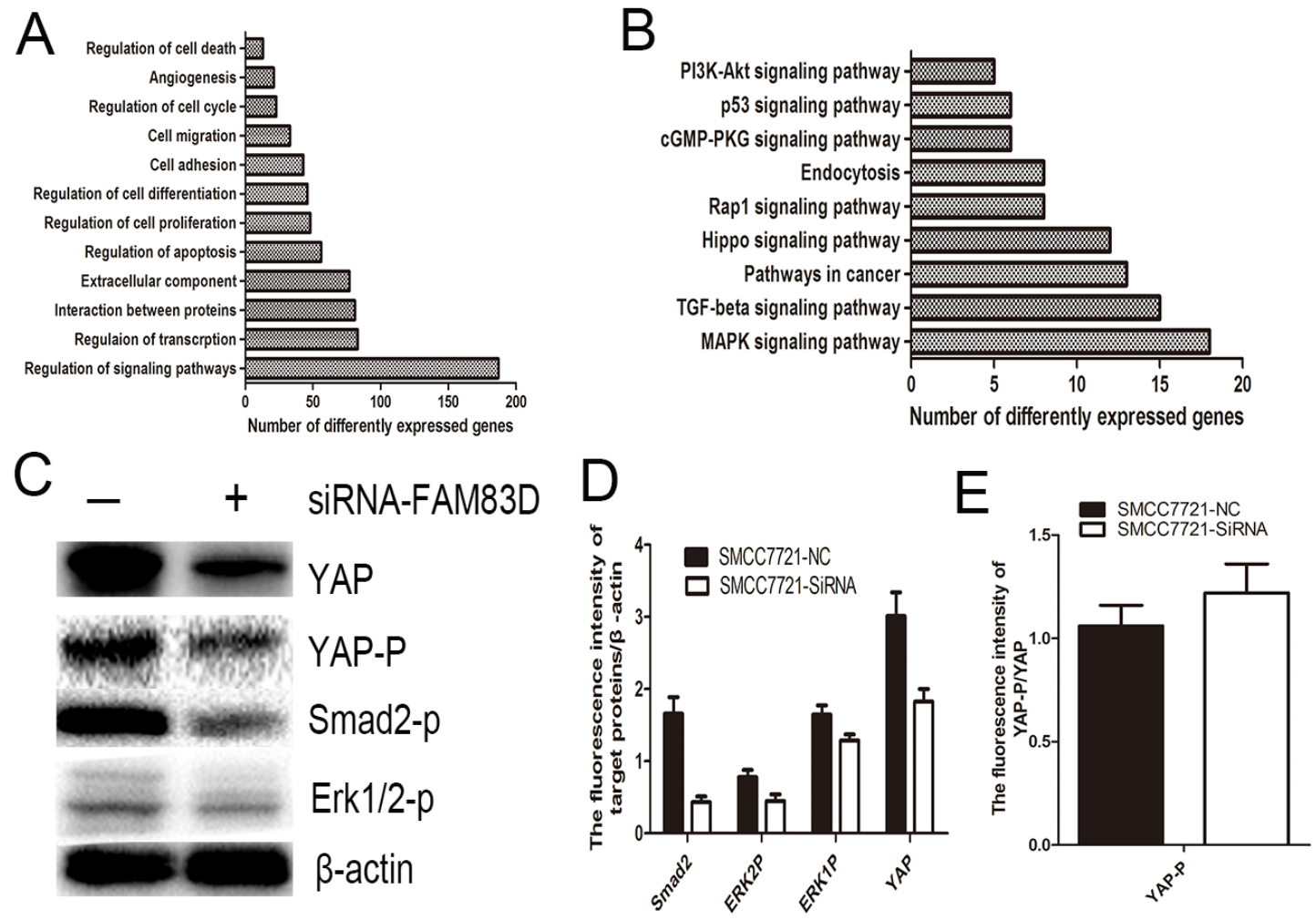

Figure 6: Gene expression microarray provided a total of 1565 differentially expressed genes. The Kyoto Encyclopedia of Genes and Genomes (KEGG) analysis and gene ontology analysis revealed that FAM83D regulated multiple biological process such as cell migration, differentiation, adhesion A., and multiple CSCs associated signaling pathways including TGF- $\beta$, MAPK, Hippo signaling pathways B. FAM83D knockdown reduced the levels of smad2 phosphorylation, ERK1/2 phosphorylation, decreased the YAP expression C, D. and promoted the proportion of phosphorylated YAP E. 
and promoted the proportion of phosphorylated YAP (Figure 6C-6E). The results suggested that FAM83D promoted CD44 expression via activating TGF- $\beta$, MAPK and Hippo signaling pathways.

\section{The inhibitors of MAPK, Hippo and TGF-beta signaling pathways suppress CD44 expression}

To further explore the effect of MAPK, Hippo and TGF-beta signaling pathways on CD44 expression, the SCH772984 (an inhibitor of ERK1/2 phosphorylation), SB505124 (an inhibitor of smad2/3 phosphorylation) and Verteporfin [25] (an inhibitor of YAP-TEAD interaction) were respectively used to inhibit corresponding signaling pathways (Figure 7A-7C), and results suggested that Verteporfin, SCH772984 and SB505124 could significantly suppressed fluorescence intensity of CD44 expression (Figure 7D-7E). These results were further verified by western blotting (Figure 7A, 7F).

\section{CD44 is a key upstream regulator of MAPK, Hippo and TGF- $\beta$ signaling pathways}

Well known that CD44 is an importantly upstream regulator of multiple signaling pathways, the HA-CD44 or $\mathrm{HA}-\mathrm{CD} 44 \mathrm{v}$ interactions could promote activation of multiple kinases [22]. Therefore, a siRNA-CD44 transfection assay was used to investigate that the effect of CD44 knock-down on activation of MAPK, Hippo and TGF-beta signaling pathways. We found that CD44 knock-down significantly reduced the level of ERK1/2 phosphorylation, Smad2 phosphorylation and YAP expression, but increased YAP phosphorylation (Figure $8 \mathrm{~A}-8 \mathrm{C}$ ). These results suggested that CD44 is also a key upstream regulator of activating MAPK, TGF-beta and Hippo signaling pathways.

\section{The analysis of protein types interacting with FAM83D}

An online database (http://string-db.org/) was used to investigate the protein types which potentially interact with FAM83D. Combing the data from a recent published article, we found potential nine proteins as follows, PPP1R16A, PPP1R16B, SLC32A1, CSNK1E, CSNK1A1, UBC, KIF4A, KIF22, CRAF (Figure 8D). Subsequently, these proteins were further analyzed by the online database (http://david.abcc.ncifcrf.gov/). The results of pathway analysis revealed that CSNK1E, CSNK1A1 involves in Wnt and Hedgehog signaling pathway, and CRAF is a promotor of MAPK pathways, but no proteins could regulates Hippo and TGF- $\beta$ signaling pathways. These results potentially suggested that FAM83D activated MAPK signaling pathway via transferring location of CRAF in membrane leaded to decreased CD44 expression, and CD44 reduction inhibited TGF- $\beta$ and Hippo signaling pathways (Figure 8E).
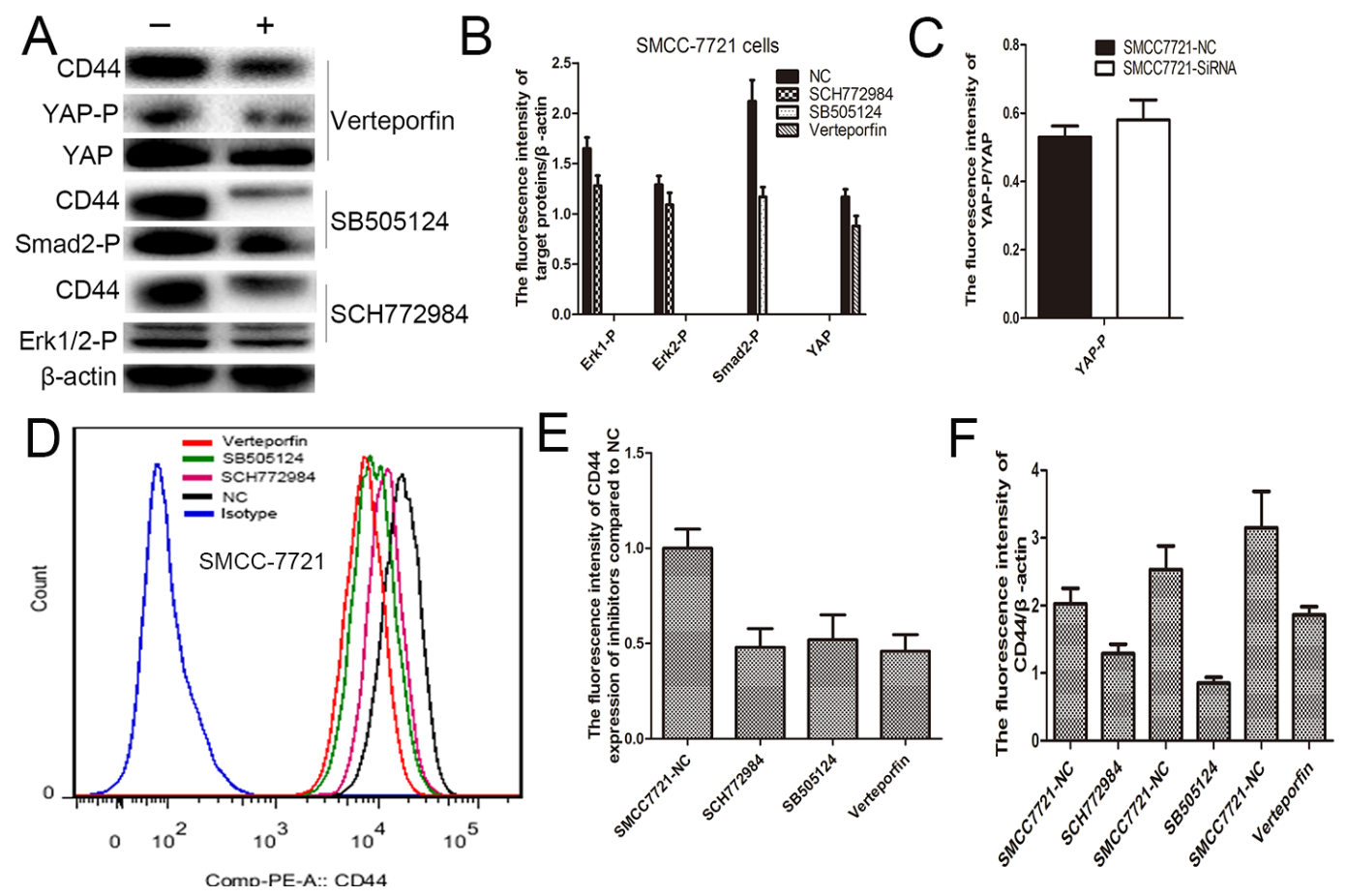

Figure 7: SCH772984, SB505124 and Verteporfin administration significantly inhibited the corresponding signaling pathways A-C. FCS confirmed that SCH772984, SB505124 and Verteporfin administration apparently reduced the CD44 expression D, E., and western blot verified this result too A, F. 


\section{DISCUSSION}

Despite the great advances in surgical and immunosuppressors, the long-term survival of LT patients for HCC remains challenging due to a high incidence of $\mathrm{HCC}$ recurrence [26]. A better understanding of the molecular mechanisms underlying HCC metastasis will promote management and prognosis. To investigate the genes involving in the complicated metastasis process, we profiled the mRNA in HCC with RNA sequence. Following the pathway analysis, of the cancer related signaling pathways, the maximum genes located in the MAPK signaling pathway. Previously published data verified that FAM83 family bind CRAF to promote activation of MAPK signaling pathway, but the role and mechanism of FAM83 family in HCC was far clarified.

In this study, we confirmed that FAM83D was significantly overexpressed in HCC tissues compared with the adjacent non tumor tissues, and correlated with high HCC recurrence following LT. The TFS of the high FAM83D expression group was apparently poorer than the low FAM83D expression group, and the difference maintained in the HCC patients transplanted no matter beyond or within Milan criteria. These results suggested that FAM83D possibly is a precise indicator of improving selection of LT candidates. Furthermore, high FAM83D expression was verified correlating with tumor size, tumor number, PVTT and AFP levels. The AFP is produced by embryonic or infantile liver cells, which reminded that FAM83D potentially regulate CSCs functions.

Growing evidences demonstrated that CSCs possess the ability of self-renewal and give rise to antitumor drug resistance and tumor relapse, metastasis resulting in disease progression and motality [27, 28]. In despite of lacking simple, rigorous assays for defining the number of CSCs [29], measuring CSCs biomarkers potentially help clinicians to relist LT candidates. Llovet $\mathrm{JM}$ et al revealed that HCC patients without CSCs markers expression transplanted beyond Milan criteria achieved TFS rates similar as HCC patients transplanted within Milan criteria [30]. We investigated the role of FAM83D in CSCs. Of all CSCs biomarkers, CD44 expression was apparently down regulated following FAM83D knockdown. This result was further confirmed by FCS. FAM83D knockdown made a decrease in proportion of CD44 positive cells and a significantly decrease in fluorescence intensity of CD44 expression. In addition, we found that CD44 expression in HCC patients with high FAM83D expression was apparently higher than those with low FAM83D expression. In addition, FAM83D knockdown inhibited the ability of sphere formation, selfrenew and differentiation. Besides, FAM83D knockdown HCC cells were injected into nude mice via tail vein or subcutaneously, and found that FAM83D knockdown inhibited the ability of tumorigenesis, pulmonary tumor colonization and tumor metastasis. These results suggested
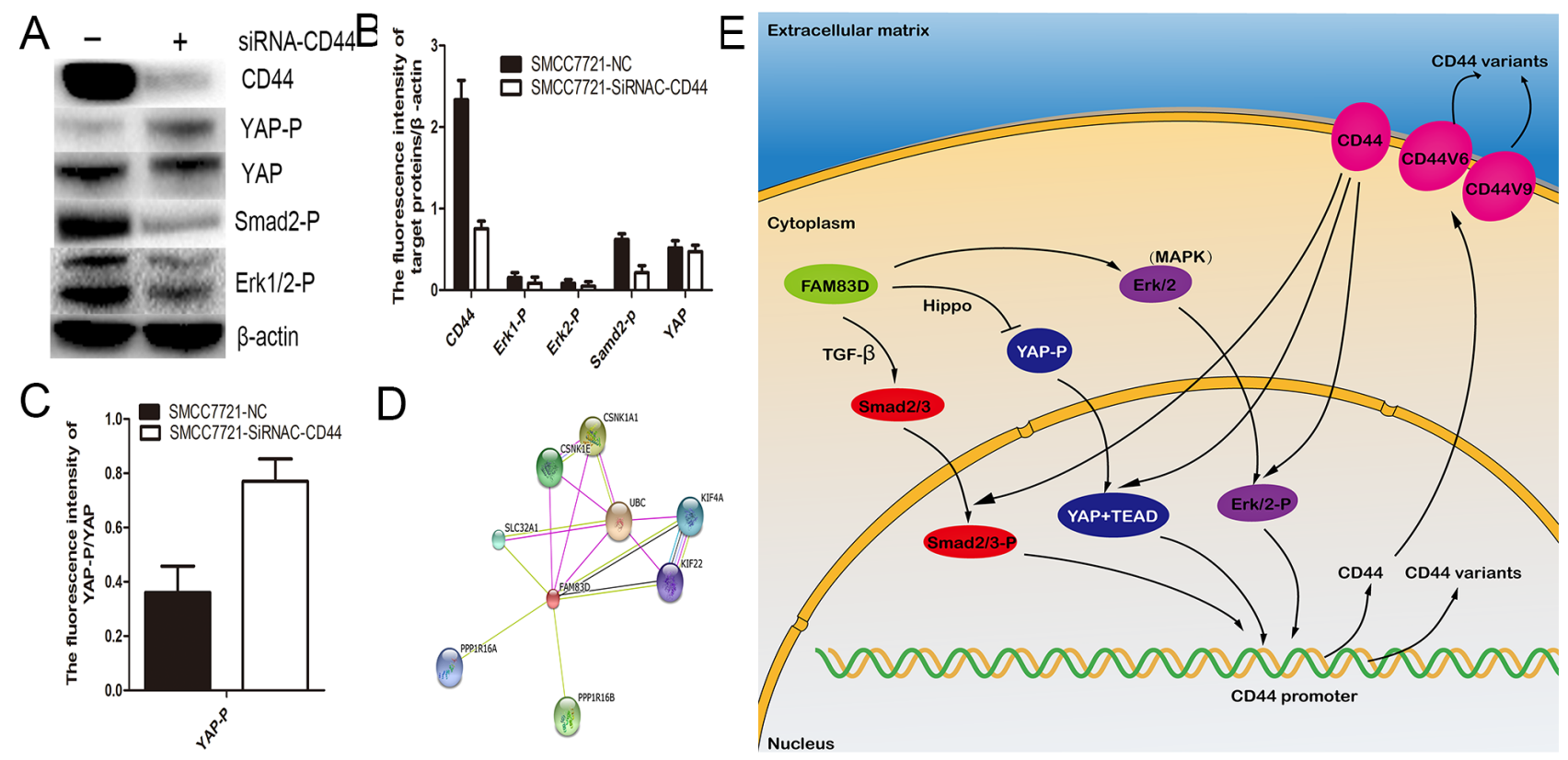

Figure 8: CD44 knockdown markedly reduced the level of ERK1/2 phosphorylation, Smad2 phosphorylation and YAP expression A, B., and increased YAP phosphorylation C. By an online database (http://string-db.org/), eight proteins including PPP1R16A, PPP1R16B, SLC32A1, CSNK1E, CSNK1A1, UBC, KIF4A, KIF22 were considered as potential protein interacting with FAM83D D. The map of molecular mechanism underlying FAM83D drives expansion of CD44 positive CSCs E. 
FAM83D is a promotor of CD44 expression and CD44 CSCs malignancy.

CD44 attracted considerable interest for its abundant expression on CSCs [31], and overexpressed in local tumor tissues promoting tumor progression [22, $32,33]$. Recently, several articles revealed that targeting CD44 positive tumor cells with anti-CD44 monoclonal antiboby apparently inhibited proliferation and promoted apoptosis of sphere forming cells [34], and improved cells uptake of other anti-tumor chemicals [35], but the efficiency was influenced by CD44 isoform status [36]. Intriguingly, FAM83D not only inhibited the standard CD44 expression, but also down regulated the CD44 variants expression. Therefore, FAM83D potentially served as a novel target for improving the effect of antiCD44 monoclonal antiboby therapy.

To explore the mechanism underlying FAM83D regulating the $\mathrm{CD} 44$ expression in $\mathrm{HCC}$, a gene expression microarray assay was carried out. FAM83D knockdown inhibited MAPK, TGF- $\beta$ and Hippo signaling pathway. Previously published data confirmed that these pathways were associated with CSCs phenotypes [37-39]. To our knowledge, only Arteaga, C. L et al revealed that DUSP4 loss promotes activation of MAPK pathways leading to increase $\mathrm{CD} 44^{+} \mathrm{CD} 24$ cells population in basal-like breast cancer [37]. Currently, the role of MAPK, TGF- $\beta$ and Hippo pathways in regulating CD44 expression in HCC was unclear. In the study, the SCH772984, SB505124 and Verteporfin were used to inhibit the corresponding signaling pathway, and we found that administrating Verteporfin, SCH772984 and SB505124 significantly inhibited the CD44 expression in HCC cell lines. These results firstly demonstrated the role of TGF- $\beta$ and Hippo pathways in promoting CD44 expression.

Well-know that HA-CD44 and HA-CD44v interactions take a central role in multiple signaling pathways especially in receptor tyrosine kinase induced activation of pathways. A few articles revealed the interaction of CD44 and TGF- $\beta$, Hippo and MAPK signaling pathways. The interaction of CD44 and TGF- $\beta$ pathways promoted breast tumor metastasis [40]. Xu Y et al demonstrated that CD44 attenuated phosphorylation level of the YAP leaded to activating Hippo pathway to enhancing efficacy of chemotherapy in glioblastoma multiforme [41]. P Zhao et al revealed that CD44 was necessary for MAPK pathways dependent lung adenocarcinoma proliferation [42]. To investigate the role of CD44 in regulating TGF- $\beta$, Hippo and MAPK signaling pathways in HCC, a siRNA-CD44 was transfected into HCC cell lines. We found that CD44 knockdown apparently activated Hippo pathway and inhibited MAPK and TGF- $\beta$ pathways. These results confirmed that CD44 is also a key upstream regulator of activating MAPK, TGF-beta and Hippo signaling pathways.

Combining the data from recent articles and an online database, we found nine proteins potentially interacting with FAM83D. Among them, CSNK1E and CSNK1A1 activate Wnt and Hedgehog signaling pathways, CRAF promotes MAPK pathways, but no one stimulate Hippo and TGF- $\beta$ signaling pathways. In the study, CD44 made a positive feedback with MAPK, Hippo and TGF-beta signaling pathways. Furthermore, FAM83D promoted the positive feedback leaded to CD44 overexpression and increased CSCs phenotype in HCC.

In summary, HCC patients with high FAM83D expression significantly correlate with the high recurrence rate of HCC following LT. The underlying mechanism involves FAM83D promoting expansion of CD44 positive CSCs via activating MAPK, Hippo and TGF-beta signaling pathways. FAM83D potentially provide a novel therapeutic approach against HCC recurrence after LT.

\section{MATERIALS AND METHODS}

\section{Clinical specimen collection}

The study was performed according to the ethical guidelines of the Declaration of Helsinki in 1975, and the experimental protocols were approved by the ethics committee of ZhangJiang University. Informed written consent was obtained from all LT patients. Samples from 150 HCC patients with LT at The First Affiliated Hospital, ZhangJiang University School of Medicine between 2006 and 2013 were collected for the study. Patients' data were followed and collected via hospital of information collection system of LT database.

\section{Cells culture}

Seven human HCC cell lines (HepG2, Hep3B, Huh-7, SK-Hep-1, SMMC-7721, MHCC97H, MHCC97L and MHCC-LM3) and one immortalized normal liver cell line $\left(\mathrm{LO}_{2}\right)$ were purchased from Cell Bank of Type Culture Collection of Chinese Academy of Sciences, Shanghai Institute of Cell Biology, Chinese Academy of Sciences and respectively cultivated according to the suppliers. HepG2, Hep3B, Huh-7, SK-Hep-1, MHCC97H, MHCC 97L and MHCC-LM3 were cultured in the Dulbecco's modified Eagle medium (DMEM; GibcoInvitrogen, Carlsbad, CA, USA) supplemented with $10 \%$ fetal bovine serum and $1 \%$ penicillin/streptomycin. SMMC-7721 and $\mathrm{LO}_{2}$ were cultured in 1640 complete medium supplemented with $10 \%$ fetal bovine serum and $1 \%$ penicillin/streptomycin. All cell lines were incubated in a humidified atmosphere of $5 \% \mathrm{CO}_{2}$ at $37^{\circ} \mathrm{C}$.

\section{Antibodies and chemicals}

FAM83D antibody (Santa Cruz Biotechnology, CA, USA), Goat anti-Mouse HRP (ABCAM, Cambridge, MA, USA), $\beta$-actin antibody, CD44 antibody, Smad2/3 
antibody, Smad2/3-P antibody, YAP/TAZ antibody, YAP-P antibody, ERK1/2 antibody and ERK1/2-P antibody, Goat anti-Rabbit HRP (Cell Signaling Technology, Beverly, MA, USA). Anti-CD44-PE, Anti-CD44-FITC (BD Pharmingen, NJ, USA). SCH772984, SB505124 and Verteporfin (Selleck Chemicals, TX, USA).

\section{Quantitative real time PCR (RT-PCR)}

Total RNA from samples was extracted with a TRIzol RNA extraction kit (Invitrogen Co.). Reverse transcription was performed with a Rever Tre Ace-areverse transcription kit (Invitrogen Co.) to synthesize complementary DNA. The RT-PCR was performed with a Roche LightCycler using Takara SYBR Premix Extaq system. All preforming procedures were followed the instructions of manufactures. Primers were generated by Shanghai Sangon Biological Engineering Technology Services Co., Ltd. The nucleotide sequences of each primer presented in supplementary Table 1.

\section{Transfections of lentiviral vectors with FAM83D siRNA}

To investigate the function of FAM83D on HCC, a FAM83D siRNA lentiviral vector (lenti-siRNA/ FAM83D) was constructed (Shanghai GeneChem Co., Ltd., Shanghai, China), as well as a GFP-lentiviral vector with a scramble sequence (Scr-siRNA/GFP) as a negative control. Untreated SMMC-7721 cell and SK-Hep-1 cell at $5 * 105$ were respectively plated into six-well plates for $24 \mathrm{~h}$. Monolayer cells per well with $20-30 \%$ confluency were transfected with lentiviral of lenti-siRNA/FAM83D or Scr-siRNA according to the manufacturer's instructions. To establish the stable cell lines, transfected cells were selected with $4 \mathrm{mg} / \mathrm{ml}$ of puromycin.

\section{Western blotting}

Cells were collected and lysed in lysis buffer (Cell Signaling Technology, Beverly, MA, USA) at $4{ }^{\circ} \mathrm{C}$ following the manufacturer's instructions. Western blotting analysis was performed according to the standard protocol. Briefly, protein concentrations were defined by a Bradford assay (Bio-Rad, Hercules, CA, USA) with a BCA Protein Assay Kit (Pierce, Rockford, IL, USA). Equal amounts of protein were separated by SDS-PAGE and then electrophoretically transferred to the $0.45 \mathrm{um}$ polyvinylidene fluoride (PVDF) membrane (Millipore, Bedford, MA, USA). The membrane was soaked and blocked with 5\% nonfat milk in Trisbuffered saline supplied with $0.05 \%$ Tween-20 (TBST) at room temperature. After washing three times with TBST, membrane was incubated with the target primary antibodies (1:1000) overnight at $4^{\circ} \mathrm{C}$, and experienced second washing and then incubated with the second corresponding antibodies (1:2000) at room temperature for 1 hour. The protein expression was visualized by SuperSignal West Pico Chemiluminescent Substrate (Pierce, Billerica, MA, USA).

\section{Immunohistochemistry}

Immunohistochemical analysis of paired paraffinembedded HCC tissues and adjacent normal tissues was carried out. Briefly, after experienced deparaffinating and rehydration, serial $4 \mu \mathrm{m}$ sections then acquired heatinduced epitope retrieval. Next, 3\% hydrogen peroxide was used to quench the endogenous peroxidase activity, and 5\% FBS was used to block nonspecific binding. After blocking, the sections were incubated with primary monoclonal antibodies overnight at $4^{\circ} \mathrm{C}$. The sections were incubated with secondary antibodies conjugatedHRP for $1 \mathrm{~h}$ at room temperature and then visualized by 3,3'-diaminobenzidine, and followed by counterstaining with hematoxylin. Furthermore, phosphate-buffered saline replaced the primary antibody (PBS) to establish the negative control.

\section{Flow cytometry analysis}

FAM83D-siRNA transfected cells or SCH772984, SB505124 and verteporfin treated cells were tested for the surface markers CD44 by incubating with anti-CD44-PE. Isotype controls were used to determine the background flurecscence. After washing second three times with PBS, CD44 expression was read in a 4-color FC500 flow cytometer (Beckman-Coulter, Miami, FL) for $1 * 10^{4}$ cellular events.

\section{Tumorsphere formation assay}

To testing growth of primary tumorsphere, 1000 cells (Control cells, FAM83D-siRNA transfected cells) were seeded in 6-well plates with ultralow attachment (Corning, Lowell, MA, USA) and grown in serum-free 1640 medium or tumorsphere forming 1640 medium supplied with serum-free DMEM/F12 supplemented with human epidermal growth factor, insulin and B27 (GibcoLife Technologies, Carlsbad, CA). The size and number of tumorsphere were analyzed in culture using an inverted microscope after 7-10 days.

\section{Tumor xenograft experiment}

All the experiments procedures were performed in accordance with the guidelines for the National Institutes of Health guide for the care and use of laboratory animals. Firstly, 5*106 cells/mice were injected subcutaneously in the lateral flank of immunodeficient mice. Tumor volume (measured weekly with a caliper) was calculated using the equation: Tumor size $=\left(\right.$ Width $^{2}$ *Length)/2. After 4 weeks, half of Mice were sacrificed and tumors were harvested for immunostaining after 
cell injection, and other half of mice were sacrificed and defined the number of lung metastasis after 6 weeks. Furthermore, for testing the ability of tumor colony in lung, $1 * 10^{6}$ cells/mice were injected into immunodeficient mice via tail vein. After 6 weeks, Mice were sacrificed and the numbers of tumor colonies in lung were analyzed by haematoxylin eosin staining.

\section{Cell migration and invasion assays}

For the transwell migration assays, $3 * 10^{4} \mathrm{SMMC}$ 7721 cells in serum-free medium were plated in the upper chamber with a non-coated membrane (24-well insert; 8-mm pore size; Millipore, Billerica, MA, USA). For invasion assays, $3 * 10^{4} \mathrm{SMMC}-7721$ cells in serumfree medium were placed in the upper chamber with a Matrigelcoated membrane. In both assays, the lower chamber were filled with 500ul culture medium supplied with $10 \%$ FBS. After incubation for $24 \mathrm{~h}, 48 \mathrm{~h}$ and $72 \mathrm{~h}$ (migration and invasion assay), cells on the lower membrane were counted by fixed with $95 \%$ methanol and stained with $0.2 \%$ crystal violet.

\section{Statistical analysis}

All of experiments were performed thrice and the data were presented as mean $\pm \mathrm{SD}$ or frequency. A two-tailed Student's t test and Fisher's exact test were performed to analyze the potential association between FAM83D and clinicopathologic parameters, malignant phenotype. Tumor free survival (TFS) was calculated from the date of LT until HCC recurrence. Potential predictive factors were analyzed by Kaplan-Meier curves and the log-rank test. Preoperative factors with significance $(\mathrm{p}<$ $0.10)$ for TFS in the univariate analysis were further selected into the Cox proportional hazards model (backward selection likelihood function) to determine their independent effects. SPSS19.0 software was used for statistical analysis and $\mathrm{P}<0.05$ was considered as statistical significance.

\section{ACKNOWLEDGMENTS}

We gratefully acknowledge Lin Zhang contributing to maintaining the update of liver transplantation data bank of First Affiliated Hospital, School of Medicine, Zhejiang University.

\section{GRANT SUPPORT}

This work was supported by National Natural Science Foundation of China (No.81302074, 81373160, 81400673 ) and Zhejiang provincial natural science foundation of China (No.LQ13H160004, LY15H160016, LQ15H030003) and the medical and health plan of Zhejiang province (201472813).

\section{CONFLICTS OF INTEREST}

All authors declared that they have nothing to disclose regarding funding or conflict of interest with respect to this manuscript.

\section{REFERENCES}

1. Altekruse SF, McGlynn KA, Reichman ME. Hepatocellular carcinoma incidence, mortality, and survival trends in the United States from 1975 to 2005. Journal of clinical oncology. 2009; 27:1485-1491.

2. El-Serag HB. Epidemiology of viral hepatitis and hepatocellular carcinoma. Gastroenterology. 2012; 142:1264-1273 e1261.

3. El-Serag HB, Rudolph KL. Hepatocellular carcinoma: epidemiology and molecular carcinogenesis. Gastroenterology. 2007; 132:2557-2576.

4. Llovet JM, Ricci S, Mazzaferro V, Hilgard P, Gane E, Blanc JF, de Oliveira AC, Santoro A, Raoul JL, Forner A, Schwartz M, Porta C, Zeuzem S, et al. Sorafenib in advanced hepatocellular carcinoma. The New England journal of medicine. 2008; 359:378-390.

5. Postma C, Terwischa S, Hermsen MA, van der Sijp JR, Meijer GA. Gain of chromosome 20q is an indicator of poor prognosis in colorectal cancer. Cellular oncology. 2007; 29:73-75.

6. Inamura $\mathrm{K}$, Shimoji $\mathrm{T}$, Ninomiya $\mathrm{H}$, Hiramatsu $\mathrm{M}$, Okui M, Satoh Y, Okumura S, Nakagawa K, Noda T, Fukayama M, Ishikawa Y. A metastatic signature in entire lung adenocarcinomas irrespective of morphological heterogeneity. Human pathology. 2007; 38:702-709.

7. Ramakrishna M, Williams LH, Boyle SE, Bearfoot JL, Sridhar A, Speed TP, Gorringe KL, Campbell IG. Identification of candidate growth promoting genes in ovarian cancer through integrated copy number and expression analysis. PloS one. 2010; 5:e9983.

8. Wang Z, Liu Y, Zhang P, Zhang W, Wang W, Curr K, Wei G, Mao JH. FAM83D promotes cell proliferation and motility by downregulating tumor suppressor gene FBXW7. Oncotarget. 2013; 4:2476-2486. doi: 10.18632/ oncotarget.1581.

9. Sauer G, Korner R, Hanisch A, Ries A, Nigg EA, Sillje HH. Proteome analysis of the human mitotic spindle. Molecular \& cellular proteomics: MCP. 2005; 4:35-43.

10. Santamaria A, Nagel S, Sillje HH, Nigg EA. The spindle protein CHICA mediates localization of the chromokinesin Kid to the mitotic spindle. Current biology: CB. 2008; 18:723-729.

11. Cipriano R, Graham J, Miskimen KL, Bryson BL, Bruntz RC, Scott SA, Brown HA, Stark GR, Jackson MW. FAM83B mediates EGFR- and RAS-driven oncogenic transformation. The Journal of clinical investigation. 2012; 122:3197-3210. 
12. Cipriano R, Miskimen KL, Bryson BL, Foy CR, Bartel CA, Jackson MW. Conserved oncogenic behavior of the FAM83 family regulates MAPK signaling in human cancer. Molecular cancer research: MCR. 2014; 12:1156-1165.

13. Dalerba P, Cho RW, Clarke MF. Cancer stem cells: models and concepts. Annual review of medicine. 2007; 58:267-284.

14. Hermann PC, Huber SL, Herrler T, Aicher A, Ellwart JW, Guba M, Bruns CJ, Heeschen C. Distinct populations of cancer stem cells determine tumor growth and metastatic activity in human pancreatic cancer. Cell stem cell. 2007; 1:313-323.

15. Zeng Z, Ren J, O'Neil M, Zhao J, Bridges B, Cox J, Abdulkarim B, Schmitt TM, Kumer SC, Weinman SA. Impact of stem cell marker expression on recurrence of TACE-treated hepatocellular carcinoma post liver transplantation. BMC cancer. 2012; 12:584.

16. Marsden CG, Wright MJ, Pochampally R, Rowan BG. Breast tumor-initiating cells isolated from patient core biopsies for study of hormone action. Methods in molecular biology (Clifton, NJ). 2009; 590:363-375.

17. Lee TK, Castilho A, Cheung VC, Tang KH, Ma S, Ng IO. $\mathrm{CD} 24(+)$ liver tumor-initiating cells drive self-renewal and tumor initiation through STAT3-mediated NANOG regulation. Cell stem cell. 2011; 9:50-63.

18. Ma S, Chan KW, Hu L, Lee TK, Wo JY, Ng IO, Zheng BJ, Guan XY. Identification and characterization of tumorigenic liver cancer stem/progenitor cells. Gastroenterology. 2007; 132:2542-2556.

19. Haraguchi N, Ishii H, Mimori K, Tanaka F, Ohkuma M, Kim HM, Akita H, Takiuchi D, Hatano H, Nagano H, Barnard GF, Doki Y, Mori M. CD13 is a therapeutic target in human liver cancer stem cells. The Journal of clinical investigation. 2010; 120:3326-3339.

20. Yamashita T, Ji J, Budhu A, Forgues M, Yang W, Wang HY, Jia H, Ye Q, Qin LX, Wauthier E, Reid LM, Minato H, Honda M, Kaneko S, Tang ZY, Wang XW. EpCAMpositive hepatocellular carcinoma cells are tumor-initiating cells with stem/progenitor cell features. Gastroenterology. 2009; 136:1012-1024.

21. Yang ZF, Ho DW, Ng MN, Lau CK, Yu WC, Ngai P, Chu PW, Lam CT, Poon RT, Fan ST. Significance of CD90+ cancer stem cells in human liver cancer. Cancer Cell. 2008; 13:153-166.

22. Zoller M. CD44: can a cancer-initiating cell profit from an abundantly expressed molecule? Nature reviews Cancer. 2011; 11:254-267.

23. Oishi N, Yamashita T, Kaneko S. Molecular biology of liver cancer stem cells. Liver cancer. 2014; 3:71-84.

24. Huang da W, Sherman BT, Lempicki RA. Systematic and integrative analysis of large gene lists using DAVID bioinformatics resources. Nature protocols. 2009; 4:44-57.

25. Liu-Chittenden Y, Huang B, Shim JS, Chen Q, Lee SJ, Anders RA, Liu JO, Pan D. Genetic and pharmacological disruption of the TEAD-YAP complex suppresses the oncogenic activity of YAP. Genes \& development. 2012; 26:1300-1305.

26. Befeler AS, Hayashi PH, Di Bisceglie AM. Liver transplantation for hepatocellular carcinoma. Gastroenterology. 2005; 128:1752-1764.

27. Tovar V, Cornella H, Moeini A, Vidal S, Hoshida Y, Sia D, Peix J, Cabellos L, Alsinet C, Torrecilla S, MartinezQuetglas I, Lozano JJ, Desbois-Mouthon C, et al. Tumour initiating cells and IGF/FGF signalling contribute to sorafenib resistance in hepatocellular carcinoma. Gut. 2015.

28. Vidal SJ, Rodriguez-Bravo V, Galsky M, Cordon-Cardo C, Domingo-Domenech J. Targeting cancer stem cells to suppress acquired chemotherapy resistance. Oncogene. 2014; 33:4451-4463.

29. Kaiser J. The cancer stem cell gamble. Science (New York, NY). 2015; 347:226-229.

30. Miltiadous O, Sia D, Hoshida Y, Fiel MI, Harrington AN, Thung SN, Tan PS, Dong H, Revill K, Chang CY, Roayaie S, Byrne TJ, Mazzaferro V, et al. Progenitor cell markers predict outcome of patients with hepatocellular carcinoma beyond Milan criteria undergoing liver transplantation. Journal of hepatology. 2015; 63:1368-1377.

31. Ratajczak MZ. Cancer stem cells--normal stem cells "Jedi" that went over to the "dark side". Folia histochemica et cytobiologica/Polish Academy of Sciences, Polish Histochemical and Cytochemical Society. 2005; 43:175-181.

32. Kakehashi A, Ishii N, Sugihara E, Gi M, Saya H, Wanibuchi H. CD44v9 is a potential biomarker of tumor initiating cells predicting survival outcome in $\mathrm{HCV}$-positive patients with resected HCC. Cancer science. 2016.

33. Yan Y, Li Z, Kong X, Jia Z, Zuo X, Gagea M, Huang S, Wei D, Xie K. KLF4-mediated Suppression of CD44 Signaling Negatively Impacts Pancreatic Cancer Stemness and Metastasis. Cancer research. 2016.

34. Du YR, Chen Y, Gao Y, Niu XL, Li YJ, Deng WM. Effects and mechanisms of anti-CD44 monoclonal antibody A3D8 on proliferation and apoptosis of sphere-forming cells with stemness from human ovarian cancer. International journal of gynecological cancer. 2013; 23:1367-1375.

35. Arabi L, Badiee A, Mosaffa F, Jaafari MR. Targeting CD44 expressing cancer cells with anti-CD44 monoclonal antibody improves cellular uptake and antitumor efficacy of liposomal doxorubicin. Journal of controlled release. 2015; 220:275-286.

36. Birzele F, Voss E, Nopora A, Honold K, Heil F, Lohmann $\mathrm{S}$, Verheul H, Le Tourneau C, Delord JP, van Herpen C, Mahalingam D, Coveler AL, Meresse V, Weigand S, Runza V, Cannarile M. CD44 Isoform Status Predicts Response to Treatment with Anti-CD44 Antibody in Cancer Patients. Clinical cancer research. 2015; 21:2753-2762.

37. Balko JM, Schwarz LJ, Bhola NE, Kurupi R, Owens P, Miller TW, Gomez H, Cook RS, Arteaga CL. Activation 
of MAPK pathways due to DUSP4 loss promotes cancer stem cell-like phenotypes in basal-like breast cancer. Cancer research. 2013; 73:6346-6358.

38. Wang XH, Liu MN, Sun X, Xu CH, Liu J, Chen J, Xu RL, Li BX. TGF-betal pathway affects the protein expression of many signaling pathways, markers of liver cancer stem cells, cytokeratins, and TERT in liver cancer HepG cells. Tumour biology: the journal of the International Society for Oncodevelopmental Biology and Medicine. 2015.

39. Nandy SB, Arumugam A, Subramani R, Pedroza D, Hernandez K, Saltzstein E, Lakshmanaswamy R. MicroRNA-125a influences breast cancer stem cells by targeting leukemia inhibitory factor receptor which regulates the Hippo signaling pathway. Oncotarget. 2015; 6:17366-17378. doi: 10.18632/oncotarget.3953.
40. Bourguignon LY, Singleton PA, Zhu H, Zhou B. Hyaluronan promotes signaling interaction between CD44 and the transforming growth factor beta receptor I in metastatic breast tumor cells. The Journal of biological chemistry. 2002; 277:39703-39712.

41. Xu Y, Stamenkovic I, Yu Q. CD44 attenuates activation of the hippo signaling pathway and is a prime therapeutic target for glioblastoma. Cancer research. 2010; 70:2455-2464.

42. Zhao P, Damerow MS, Stern P, Liu AH, Sweet-Cordero A, Siziopikou K, Neilson JR, Sharp PA, Cheng C. CD44 promotes Kras-dependent lung adenocarcinoma. Oncogene. 2013; 32:5186-5190. 